\title{
Uncertainty Characteristics of Subjective Opinions
}

\author{
Audun Jøsang \\ University of Oslo \\ Oslo, Norway \\ josang@mn.uio.no
}

\author{
Jin-Hee Cho \\ Army Research Laboratory \\ Adelphi, MD, USA \\ jin-hee.cho.civ@mail.mil
}

\author{
Feng Chen \\ SUNY-Albany \\ Albany, NY, USA \\ fchen5ealbany.edu
}

\begin{abstract}
In this work, we study different types of uncertainty in subjective opinions based on the internal belief mass distribution and the base rate distribution. Subjective opinions which are used as arguments in subjective logic (SL) expand the traditional belief functions by including base rate distributions. Fundamental uncertainty characteristics of a given opinion depend on its 'singularity', 'vagueness', 'vacuity', 'dissonance', 'consonance' and 'monosonance'. We define those concepts in the formalism of SL and show how these characteristics can be manifested in the three different opinion classes which are binomial, multinomial, and hyper-opinions. We clarify the relationships between the uncertainty characteristics and discuss how they influence decision making in SL.
\end{abstract}

\section{INTRODUCTION}

In all sorts of business processes as well as in our private life, we are confronted with various kinds of decisions involving multiple choices and relative uncertainty. A clear understanding of the uncertainty is a prerequisite of sound and effective decision making. Although the topic of reasoning under uncertainty is well established and has been studied since the 1960s, many belief and reasoning models are unable to represent uncertainty in terms of its different manifestations. The era of the Internet and Big Data brings with it a flood of information which can be leveraged for decision making. In this situation, the challenge for timely and accurate decision making is no longer the lack of information, but the risk of not being able to understand and manage its inherent uncertainty resulting from unreliable, incomplete, deceptive, and conflicting information.

Evidence or belief theories have been popularly used in decision making under uncertainty since the 1960s, including Fuzzy Logic [18], Dempster-Shafer Theory (DST) [11] and Probabilistic Soft Logic (PSL) [6, 8]. These models all have in common that they offer a richer expression of uncertainty and fuzziness than the traditional propositional logic or probability calculus can offer.

Subjective logic (SL) explicitly represents second-order probabilistic uncertainty through a subjective opinion which consists of a belief mass distribution, a base rate distribution, and uncertainty mass [7]. Subjective opinions implicitly also represent other uncertainty characteristics which is the topic of the present study.

\footnotetext{
* In the proceedings of the 21th International Conference on Information Fusion (FUSION 2018), Cambridge, July, 2018.
}

In the present work, we analyze the internal belief-mass structure of subjective opinions in order to identify various uncertainty characteristics that can be relevant for visualization, understanding, reasoning and decision making. To be specific, this work has the following key contributions:

1) We define fundamental uncertainty characteristics of subjective opinions in terms of singularity, vagueness, vacuity, dissonance, consonance and monosonance. In addition, we define sharpness and bluntness of vague belief mass. Although the concepts of singularity, vacuity and vagueness are well defined in SL, dissonance, consonance, and monosonance as well as sharpness and bluntness of vague belief mass have not been investigated previously. To the best of our knowledge, this work is the first to clarify how to consider all these characteristics of uncertainty in subjective opinions.

2) We propose a simple visualization technique which shows the relationships between the various uncertainty aspects of an opinion. To this end, we examined belief mass distributions $\left(\boldsymbol{b}_{X}\right)$, uncertainty mass $\left(u_{X}\right)$, base rate distributions $\left(\boldsymbol{a}_{X}\right)$, projected probability distributions $\left(\mathbf{P}_{X}\right)$, singularity, vagueness, dissonance, consonance and monosonance for a given opinion $\omega_{X}$ on a given domain $\mathbb{X}$, where the opinion can be a binomial opinion, a multinomial opinion, or a hyper-opinion.

\section{BACKGROUND}

\section{A. Concept and Properties of Uncertainty}

The assessment and analysis of a specific situation can be affected by different types of uncertainty. Underlying uncertainty already starts from how to view and model a given part of the world which we call a domain. Domains are the abstract representations of states of the world, where analysts or decision makers can have beliefs about the true states of a domain. Beliefs about domains can easily be biased by an analyst or decision maker (often called "framing effect") $[15,16]$, which can cause subjective beliefs about the world to deviate from ground truth of the world (e.g., past or future events) [16]. The way a situation is formally modeled (i.e., expression of domains with sets of possible state values) can also affect the types and levels of uncertainty that a decision maker can assess and use in decision making.

Some root causes of uncertainty have been discussed from various angles in the literature $[1,15,16]$. It is natural to 
classify singularity, vagueness, and vacuity under the category called 'basic belief uncertainty' because these aspects are related through the additivity equation. Similarly, it is natural to classify consonance, dissonance, and monosonance under the category called 'intra-belief uncertainty' because these aspects are also related through a simple additivity equation. 'Basic belief uncertainty' relates to each belief/uncertainty mass in itself, where these components add up to 1 which means that they are additive. 'Intra-belief uncertainty' relates to the mutual relationships between the various belief masses as a function of how their focal state values (i.e., whether the values are isolated or overlapping), and it can be shown that there is additivity between the types of intra-uncertainty types. To be specific, we can identify the following categories of uncertainty in subjective opinions:

1) Basic Belief Uncertainty: Basic belief uncertainty results from specific aspects of each belief mass in isolation.

- Belief singularity refers to belief mass that applies exclusively to singleton state values of the domain. Singular belief reduces uncertainty because it supports specific state values of the domain. However, when singular belief applies to separate state values, it reflects dissonant belief, and thereby uncertainty as described under belief dissonance below.

- Belief vagueness results from evidence that fails to discriminate between singletons (i.e., specific state values of a domain variable) [4]. When the received evidence can be interpreted to support multiple values in a domain, or there is confusion between domain values because of their similarity, a level of vagueness can be perceived when trying to discriminate between them. Tuggy [14] explains vagueness in a linguistic context by defining it as "two or more meanings associated with a given phonological form" that can introduce "non-distinguished subcases of a single, more general meaning.' A simple example is when a color-blind person is unable to distinguish between red and green (subcases) when picking colored balls (general case) from an urn. The person can, for example, observe the ball as "red or green, but certainly not black or white."

- Belief vacuity (or lack of information or knowledge) is due to insufficient or unreliable information received from sources (e.g., vacuous belief due to unavailable information or sensor malfunction), but also limited prior knowledge (i.e., framing effect due to limited understanding of the dynamics and causality relationships of a situation) [16]. Belief vacuity is represented by 'uncertainty mass' in SL.

2) Intra-Belief Uncertainty: Intra-belief uncertainty results from specific aspects of the relationships between belief masses and uncertainty mass in an opinion.

- Belief dissonance reflects the situation when an analyst holds simultaneous contradicting beliefs about a given domain. Belief is said to be dissonant when it is based on separate evidence about different non-overlapping singleton or composite values, resulting in contradicting belief. This kind of uncertainty is caused by valid but conflicting evidence derived from the observation of spatial or temporal dynamics of a situation or from intended deception by adversarial entities (i.e., providing opposite evidence). High dissonance often results in high unpredictability [1, 15]. For example, Alice and Bob represent sources for observing and reporting the colors of balls picked from an urn. Suppose that the analyst receives advice from Alice that the color is $A=\{$ red,blue $\}$ and from Bob that the color is $B=\{$ green, black $\}$. It can be seen that the belief masses resulting from Alice and Bob's advice do not identify the exact color of the ball, hence they are vague. It can also be seen that the (composite) focal state values $A$ and $B$ do not overlap with each other as $A \cap B=\emptyset$, which means that the belief masses on $A$ and $B$ are dissonant.

- Belief consonance requires the target domain to have three or more possible state values, so that it is possible to form (composite) subsets of state values that contain other subsets, and the other in turn can contain other subsets, etc. Belief is said to be consonant when it is based on evidence about distinct subsets contained within each other. That is, while belief might support different subsets (and thereby be uncertain), there is no contradicting belief. For example, suppose Alice, Bob, and Carl report the color of a ball picked from an urn such as $A=\{$ red,blue,green $\}, B=\{$ red,blue $\}$, and $C=\{$ red $\}$, respectively. The resulting belief masses are consonant because the respective focal (composite) values are such that $C \subset B \subset \mathrm{A}$.

- Belief monosonance exists when the opinion only contains one belief mass, or only one dominant belief mass.

In the literature on probabilistic uncertainty [7, 9], two types of uncertainty are frequently discussed and distinguished:

- Aleatory uncertainty: This type of uncertainty denotes statistical uncertainty about the long term relative frequencies of possible outcomes [7]. For example, if we do not know whether a dice is loaded - and thereby unfair - then we are faced with aleatory uncertainty. This uncertainty can be reduced by throwing the dice sufficiently many times in order to estimate the long term relative frequency of each of the six possible outcomes. Therefore, aleatory uncertainty is fundamentally related to the nature of randomness [9].

- Epistemic uncertainty: This type of uncertainty is also called systematic uncertainty which refers to the fact that the outcome of a specific future or past event can be known, but there is insufficient evidence to support it. A typical example is the assassination of President Kennedy in 1963 [7], where the uncertainty is about whether he was killed by Lee Harvey Oswald and who organized it. The nature of epistemic uncertainty derives from the lack of knowledge (or data); therefore, the epistemic uncertainty can be reduced by more evidence, 
more advanced technology, and/or scientific principles to interpret the evidence (e.g., criminal investigation based on forensic science) [9].

Although uncertainty has been studied with regard to decision making in probability theory [4], belief theory $[11,12,13$, 18], possibility theory [3], and decision theory [10], there has been relatively little focus on studying the internal structure of belief and uncertainty in subjective opinions.

\section{B. Previous Work on Uncertainty Representation in SL}

Subjective Logic (SL) is a framework for reasoning under uncertainty, where situations are modeled in terms of (random) variables in the same way as e.g., Bayesian networks. SL incorporates elements from both probability models and logic, and offers a rich set of operators to infer new opinions based on input argument opinions [7]. Although the literature on SL considers the effect of vacuity and vagueness on decision making, the literature has not considered how dissonance and consonance affect decisions. Wang and Singh [17] proposed dissonance (in their terminology called ambiguity) as a characteristic of opinions for the purpose of representing belief inconclusiveness. Very recently, Cho et al. [2] also proposed an operator to deal with inconclusiveness over a belief and a disbelief in a binomial opinion where the amount of opinions supporting two extremes are about the same by lowering down uncertainty mass in SL. However, both works above [2, 17] are not only limited to binomial opinions, they also handle a reduced set of aspects of uncertainty related to subjective opinions.

Section III below explains the formal representation of subjective opinions. Then in Section IV, we discuss how the uncertainty characteristics (i.e., basic belief uncertainty and intra-belief uncertainty) can be interpreted within the formalism of SL.

\section{OPINIONS IN SL}

The type of opinion (or beliefs) depends on the random variable it applies to, i.e. binomial opinions for binary variables, multinomial opinions for random variables, and hyper-opinions for hypervariables. In this section, to be selfcontained, we provide a brief description on how each opinion type is represented in SL and what statistical distribution is used to describe each opinion type based on [7].

\section{A. Binomial Opinions}

1) Binomial Opinion Representation: In SL, a binomial opinion on a given proposition $x$ is represented by $\omega_{x}=$ $\left(b_{x}, d_{x}, u_{x}, a_{x}\right)$ where the opinion applies to the value $x$ in the binary domain $\mathbb{X}=\{x, \bar{x}\}$ and the additivity requirement of $\omega_{x}$ is given as $b_{x}+d_{x}+u_{x}=1$. To be specific, each parameter indicates,

- $b_{x}$ : belief mass supporting $x$ being TRUE;

- $d_{x}$ : disbelief mass supporting $x$ being FALSE, i.e., $\bar{x}$ being TRUE;

- $u_{x}$ : uncertainty mass representing vacuity of evidence about which value in $\mathbb{X}$ is TRUE; and
- $a_{x}$ : base rate representing a prior probability of $x$ being TRUE.

A binomial opinion can be often used for judging true vs. false or agree vs. disagree. The projected probability supporting $x$ is represented by:

$$
\mathrm{P}(x)=b_{x}+a_{x} u_{x}
$$

2) Binomial Beta Distribution: A binomial opinion is the same as a Beta PDF (probability density function) through a specific bijective mapping. Given the binary domain $\mathbb{X}=$ $\{x, \bar{x}\}$ and the value $x \in \mathbb{X}$, $\operatorname{Beta}\left(p_{x}\right)$ is the probability density function $\operatorname{Beta}\left(p_{x} ; \alpha, \beta\right):[0,1] \rightarrow \mathbb{R}_{\geq 0}$ where $p_{x}+p_{\bar{x}}=1$. The Beta PDF is given by:

$$
\operatorname{Beta}\left(p_{x} ; \alpha, \beta\right)=\frac{\Gamma(\alpha+\beta)}{\Gamma(\alpha) \Gamma(\beta)}\left(p_{x}\right)^{\alpha-1}\left(1-p_{x}\right)^{\beta-1}
$$

where $\alpha>0, \beta>0, p(x) \neq 0$ if $\alpha<1$ and $p(x) \neq 1$ if $\beta<1$; and the additivity requirement should hold with $\int_{0}^{1} \operatorname{Beta}\left(p_{x} ; \alpha, \beta\right) \mathrm{d} p_{x}=1$. The $\alpha$ and $\beta$ parameters can simply be represented by the base rate $a_{x}$ and the observation evidence $\left(r_{x}, s_{x}\right)$ where $r_{x}$ is the amount of positive evidence and $s_{x}$ is the amount of negative evidence:

$$
\alpha=r_{x}+a_{x} W, \quad \beta=s_{x}+\left(1-a_{x}\right) W .
$$

$W$ is the non-informative prior weight in the absence of positive evidence $r_{x}$ or negative evidence $s_{x}$. The expected probability of the Beta PDF is given by Eq. (4):

$$
\mathrm{E}(x)=\frac{\alpha}{\alpha+\beta}=\frac{r_{x}+a_{x} W}{r_{x}+s_{x}+W}
$$

The equivalence of a binomial opinion and a Beta PDF can be achieved through the following mapping rule:

$$
b_{x}=\frac{r_{x}}{r_{x}+s_{x}+W}, d_{x}=\frac{s_{x}}{r_{x}+s_{x}+W}, u_{x}=\frac{W}{r_{x}+s_{x}+W} .
$$

\section{B. Multinomial Opinions}

1) Multinomial Opinion Representation: In SL, a multinomial opinion in a given proposition $x$ is represented by $\omega_{X}=\left(\boldsymbol{b}_{X}, u_{X}, \boldsymbol{a}_{X}\right)$ where a domain is $\mathbb{X}$ where a random variable $X \in \mathbb{X}$ and $k=\mathbb{X}>2$ and the additivity requirement of $\omega_{x}$ is given as $\sum_{x \in \mathbb{X}} \boldsymbol{b}_{X}(x)+u_{X}=1$. To be specific, each parameter indicates,

- $\boldsymbol{b}_{X}$ : belief mass distribution over $\mathbb{X}$;

- $u_{X}$ : uncertainty mass representing vacuity of evidence;

- $a_{X}$ : base rate distribution over $\mathbb{X}$.

The projected probability distribution of multinomial opinions is given by:

$$
\mathbf{P}_{X}(x)=\boldsymbol{b}_{X}(x)+\boldsymbol{a}_{X}(x) u_{X}, \quad \forall x \in \mathbb{X}
$$

2) Multinomial Dirichlet Distribution: Multinomial probability density over a domain of cardinality $k$ is represented by the $k$-dimensional Dirichlet PDF where the special case with $k=2$ is the Beta PDF as discussed above. Denote a domain of $k$ mutually disjoint values by $\mathbb{X}$ and $\alpha_{X}$ refer to the strength vector over the values of $\mathbb{X}$ and $\mathbf{p}_{X}$ the probability distribution over $\mathbb{X}$. Dirichlet PDF with $\mathbf{p}_{X}$ as $k$-dimensional variable is defined by:

$$
\operatorname{Dir}\left(\boldsymbol{p}_{X} ; \alpha_{X}\right)=\frac{\Gamma\left(\sum_{x \in \mathbb{X}} \alpha_{X}(x)\right)}{\prod_{x \in \mathbb{X}}\left(\alpha_{X}(x)\right)} \prod_{x \in \mathbb{X}} \boldsymbol{p}_{X}(x)^{\left(\alpha_{X}(x)-1\right)}
$$


where $\alpha_{X}(x) \geq 0$ and $\mathbf{p}_{X}(x) \neq 0$ if $\alpha_{X}(x)<1$. The total strength $\alpha_{X}(x)$ for each belief value $x \in \mathbb{X}$ can be given by:

$$
\alpha_{X}(x)=\boldsymbol{r}_{X}(x)+\boldsymbol{a}_{X}(x) W \text {, where } \boldsymbol{r}_{X}(x) \geq 0, \quad \forall_{x} \in \mathbb{X}
$$

where $W$ is non-informative weight representing the amount of uncertain evidence and $\boldsymbol{a}_{X}(x)$ is the base rate distribution. Given the Dirichlet PDF, the expected probability distribution over $\mathbb{X}$ can be obtained by:

$$
\mathbf{E}_{X}(x)=\frac{\alpha_{X}(x)}{\sum_{x_{i} \in \mathbb{X}} \alpha_{X}\left(x_{i}\right)}=\frac{\boldsymbol{r}_{X}(x)+\boldsymbol{a}_{X}(x) W}{W+\sum_{x_{i} \in \mathbb{X}} \boldsymbol{r}_{X}\left(x_{i}\right)}
$$

The observed evidence in the Dirichlet PDF can be mapped to the multinomial opinions as:

$$
\boldsymbol{b}_{X}(x)=\frac{\boldsymbol{r}(x)}{\sum_{x_{i} \in \mathbb{X}} \boldsymbol{r}\left(x_{i}\right)}, u_{X}=\frac{W}{\sum_{x_{i} \in \mathbb{X}} \boldsymbol{r}\left(x_{i}\right)}
$$

Note that in binomial and multinomial opinions, each opinion over $X, x$, is a singleton opinion, which does not allow any composite value in an opinion. However, in our real life, we are often confused between multiple beliefs due to cognitive limitations or environmental noises. This kind of opinions is considered by hyper-opinions as below.

\section{Hyper-opinions}

1) Hyper-opinion Representation: Hyper-opinions allow us to represent multiple choices under a specific opinion value $x$ where belief mass is allowed to be assigned to a composite value $x \in \mathscr{C}(\mathbb{X})$ consisting of a set of singleton values. Belief mass assigned to composite values $x \in \mathbb{X}$ indicates the vagueness of an opinion. Hyperdomain, denoted by $\mathscr{R}(\mathbb{X})$, is the reduced powerset of $\mathbb{X}$ which is the set of $\mathscr{P}(\mathbb{X})$ that excludes $\{\mathbb{X}\}$ and $\{\emptyset\}$. Hyperdomain can be defined by:

$$
\text { Hyperdomain : } \mathscr{R}(\mathbb{X})=\mathscr{P}(\mathbb{X}) \backslash\{\{\mathbb{X}\},\{\emptyset\}\}
$$

Given $X$ as a hyper variable in $\mathscr{R}(\mathbb{X})$, a hyper-opinion on $X$ is represented by $\omega_{X}=\left(\boldsymbol{b}_{X}, u_{X}, \boldsymbol{a}_{X}\right)$ where each opinion dimension is,

- $\boldsymbol{b}_{X}$ : belief mass distribution over $\mathscr{R}(\mathbb{X})$;

- $u_{X}$ : uncertainty mass representing vacuity of evidence;

- $a_{X}$ : base rate distribution over $\mathbb{X}$.

where $\sum_{x \in \mathscr{R}(\mathbb{X})} \boldsymbol{b}_{X}(x)+u_{X}=1$.

The projected probability distribution of a hyper-opinion can be given by:

$$
\mathbf{P}_{X}(x)=\sum_{x_{i} \in \mathscr{R}(\mathbb{X})} \boldsymbol{a}_{X}\left(x \mid x_{i}\right) \boldsymbol{b}_{X}\left(x_{i}\right)+\boldsymbol{a}_{X}(x) u_{X}
$$

where $\boldsymbol{a}_{X}\left(x \mid x_{j}\right)$ is the relative base rate, which can be obtained by:

$$
\boldsymbol{a}_{X}\left(x \mid x_{i}\right)=\frac{\boldsymbol{a}_{X}\left(x \cap x_{i}\right)}{\boldsymbol{a}_{X}\left(x_{i}\right)}, \forall x, x_{i} \in \mathscr{R}(\mathbb{X})
$$

where $\boldsymbol{a}_{X}\left(x_{i}\right) \neq 0$. Note that for the binomial and multinomial opinions, the additivity requirement is met (i.e., $\left.\sum_{x \in \mathbb{X}} \mathbf{P}_{X}(x)=1\right)$. However, for a hyperdomain, $X \in \mathscr{R}(\mathbb{X})$ which is applied for the hyper-opinion, the additivity requirement may not be met, but $\mathbf{P}_{X}(x)$ follows super-additivity (i.e., $\left.\sum_{x \in \mathscr{R}(\mathbb{X})} \mathbf{P}_{X}(x) \geq 1\right)$.
2) Dirichlet Distributions Applied to Hyper-opinions: Hyper-opinions can be represented by Dirichlet PDFs and the hyper-Dirichlet distribution [5]. To do so, we can project a hyper-opinion into a multinomial opinion. This means we approximate the hyper-opinion with a multinomial opinion, artificially assuming that for every pair of belief values, $x_{i}, x_{j} \in \mathscr{R}(\mathbb{X})$, they do not share any belief with $x_{i} \cap x_{j}=\emptyset$, implying that the overlapping of any composite beliefs are ignored. Although this may generate some inaccuracy in practice, this provides a way to convert a hyper-opinion to a multinomial opinion. This allows the Dirichlet distribution to be applied in the hyperdomain $\mathscr{R}(\mathbb{X})$ that excludes any overlapping of composite set beliefs.

Let $\mathbb{X}$ be a domain composed of $k$ mutually disjoint values where the hyperdomain $\mathscr{R}(\mathbb{X})$ has the cardinality $\kappa=\left(2^{k}-\right.$ 1). $\alpha_{X}$ is the strength vector over $\kappa$ number of $x$ 's where $x \in \mathscr{R}(\mathbb{X})$. Given the hyper-probability distribution $\boldsymbol{p}_{X}^{H}$ and $\alpha_{X}$ that are both $\kappa$-dimensional, the Dirichlet hyper-PDF over $\boldsymbol{p}_{X}^{H}$, called Dirichlet HPDF, denoted by $\operatorname{Dir}_{X}^{H}\left(\boldsymbol{p}_{X}^{H} ; \alpha_{X}(x)\right)$, is represented by:

$$
\begin{gathered}
\operatorname{Dir}_{X}^{H}\left(\boldsymbol{p}_{X}^{H} ; \alpha_{X}(x)\right)= \\
\frac{\Gamma\left(\sum_{x \in \mathscr{R}(\mathbb{X})} \alpha_{X}(x)\right)}{\prod_{x \in \mathscr{R}(\mathbb{X})} \Gamma\left(\alpha_{X}(x)\right)} \prod_{x \in \mathscr{R}(\mathbb{X})} \boldsymbol{p}_{X}^{H}(x)^{\left(\alpha_{X}(x)-1\right)}, \\
\text { where } \alpha_{X}(x) \geq 0
\end{gathered}
$$

where $\boldsymbol{p}_{X}^{H} \neq 0$ if $\alpha_{X}(x)<0 . \alpha_{X}$ is the prior, and the observation evidence can be obtained by:

$$
\begin{gathered}
\alpha_{X}(x)=\boldsymbol{r}_{X}(x)+\boldsymbol{a}_{X}(x) W, \quad \forall x \in \mathscr{R}(\mathbb{X}) \\
\text { where } \boldsymbol{r}_{X}(x) \geq 0, \quad \boldsymbol{a}_{X}(x)=\sum_{x_{j} \subseteq x, x_{j} \in X} \boldsymbol{a}\left(x_{j}\right)
\end{gathered}
$$

Let the projection from a hyper-opinion to a multinomial opinion be denoted by $\omega_{X}^{\prime}=\left(\boldsymbol{b}_{X}^{\prime}, u_{X}, \boldsymbol{a}_{X}\right)$ where $\boldsymbol{b}_{X}^{\prime}$ is a belief mass distribution over $X \in \mathbb{X}, u_{X}$ is a uncertainty mass, and $\boldsymbol{a}_{X}$ is the base rate distribution. $\boldsymbol{b}_{X}^{\prime}$ is approximated by:

$$
\boldsymbol{b}_{X}^{\prime}(x)=\sum_{x^{\prime} \in \mathscr{R}(\mathbb{X})} \boldsymbol{a}_{X}\left(x \mid x^{\prime}\right) \boldsymbol{b}_{X}\left(x^{\prime}\right)
$$

Notice that $x \in \mathbb{X}$ while $x^{\prime} \in \mathscr{R}(\mathbb{X})$. The approximation by projection of hyper-opinions to multinomial opinions removes information in the representation of opinions. The advantage is that a decision maker is able to see a particular opinion without the veil of vagueness, which facilitates a more direct and intuitive interpretation of the opinion.

The expected probability of any of the $\kappa$ values $x \in \mathbb{X}$ can be obtained by:

$$
\mathbf{E}_{X}(x)=\frac{\sum_{x_{i} \in \mathscr{R}(\mathbb{X})} \boldsymbol{a}_{X}\left(x \mid x_{i}\right) \boldsymbol{b}_{X}\left(x_{i}\right)+W \boldsymbol{a}_{X}(x)}{W+\sum_{x_{i} \in \mathscr{R}(\mathbb{X})} \boldsymbol{r}\left(x_{i}\right)}, \forall_{x} \in \mathbb{X}
$$

The above form is the generalization of a Dirichlet PDF used in Eq. (9).

Hyper-opinions can be mapped through the Dirichlet HPDF for $\forall x \in \mathscr{R}(\mathbb{X})$ as:

$$
\boldsymbol{b}_{X}(x)=\frac{\boldsymbol{r}(x)}{\sum_{x_{i} \in \mathscr{R}(\mathbb{X})} \boldsymbol{r}\left(x_{i}\right)}, u_{X}=\frac{W}{\sum_{x_{i} \in \mathscr{R}(\mathbb{X})} \boldsymbol{r}\left(x_{i}\right)}
$$


Note that $\boldsymbol{b}_{X}(x)$ can be used to derive $\boldsymbol{b}_{X}^{\prime}(x)$ in Eq. (16) which can remove the vagueness of hyper-opinions with the sacrifice of estimation accuracy in the probability of a singleton opinion.

\section{UNCERTAINTY IN BELIEFS AND OPINIONS IN SL}

In this section, we discuss how basic belief uncertainty characteristics (i.e., singularity, vagueness, and vacuity) and mutual uncertainty characteristics (i.e., dissonance, parasonance and consonance) can be represented.

\section{A. Singular Belief Mass}

Belief mass supporting a singleton value is called singular belief mass because the singular belief mass discriminates between itself and other values. Singular belief mass on a singleton $x \in \mathbb{X}$ is simply the belief mass $\boldsymbol{b}_{X}(x)$ assigned to $x$. The total singular belief denoted $b_{X}^{\mathrm{TS} S i n}$ is simply the sum of all belief masses assigned to singletons, defined as follows.

Definition 1 (Total Singular Belief Mass): Let $\mathbb{X}$ be a domain with variable $X$, and let $\omega_{X}$ be an opinion on $\mathbb{X}$. The total singular belief mass contained in the opinion $\omega_{X}$ is the function $b_{X}^{\mathrm{TSin}}: \mathbb{X} \rightarrow[0,1]$ expressed as:

$$
\text { Total singular belief mass: } b_{X}^{\mathrm{TSin}}=\sum_{x_{i} \in \mathbb{X}} \boldsymbol{b}_{X}\left(x_{i}\right) .
$$

The total singular belief mass represents the complement of the sum of belief vacuity and total vague belief mass, as described below.

\section{B. Belief Vagueness}

In Section II-A, the concept of vagueness is described as when evidence does not distinguish between singletons. In addition, in SL, as discussed in Section III-C, vagueness only appears in hyper-opinions. The composite set, denoted $\mathscr{C}(\mathbb{X})$, is the set of all composite values from the hyperdomain. Belief mass assigned to a composite value represents vague belief mass because it expresses cognitive vagueness. It does not discriminate between the singletons in the composite value, and supports the truth of multiple singletons in $\mathbb{X}$ simultaneously. In the case of binary domains, there can be no vague belief mass, because there are no composite values. In the case of hyperdomains, composite values exist, and every singleton $x \in \mathbb{X}$ is a member of multiple composite values. The vague belief mass on a value $x \in \mathscr{R}(\mathbb{X})$ is defined as the weighted sum of belief masses on the composite values of which $x$ is a member, where the weights are determined by the base rate distribution. The total amount of vague belief mass is simply the sum of belief masses on all composite values in the hyperdomain. The formal definitions of these concepts are given as below.

Definition 2 (Vague Belief Mass): Let $\mathbb{X}$ be a domain $\mathbb{X}$ with composite set $\mathscr{C}(\mathbb{X})$. Following [7], the vague belief mass on $x \in \mathscr{R}(\mathbb{X})$ is the function $\boldsymbol{b}_{X}^{\mathrm{Vag}}: \mathscr{R}(\mathbb{X}) \rightarrow[0,1]$ :

$$
\boldsymbol{b}_{X}^{\mathrm{Vag}}(x)=\sum_{\substack{x_{i} \in \mathscr{C}(\mathbb{X}) \\ x_{i} \underline{\complement} x}} \boldsymbol{a}_{X}\left(x \mid x_{i}\right) \boldsymbol{b}_{X}\left(x_{i}\right), \quad \forall x \in \mathscr{R}(\mathbb{X}) .
$$

Note that Eq. (20) not only defines vagueness of singletons $x \in \mathbb{X}$, but also defines vagueness of composite values $x \in$ $\mathscr{C}(\mathbb{X})$, i.e., of all values $x \in \mathscr{R}(\mathbb{X})$.

The total vague belief mass in an opinion $\omega_{X}$ is defined as the sum of belief masses on composite values $x \in \mathscr{C}(\mathbb{X})$.

Definition 3 (Total Vague Belief Mass): Let $\mathbb{X}$ be a domain with variable $X$, and let $\omega_{X}$ be an opinion on $\mathbb{X}$. The total vagueness contained in the opinion $\omega_{X}$ is the function $b_{X}^{\text {TVag }}$ : $\mathscr{C}(\mathbb{X}) \rightarrow[0,1]$ expressed as:

Total vague belief mass: $b_{X}^{\mathrm{TVag}}=\sum_{x \in \mathscr{C}(\mathbb{X})} \boldsymbol{b}_{X}(x)$.

An opinion $\omega_{X}$ is dogmatic and vague when $b_{X}^{\text {TVag }}=1$, and is partially vague when $0<b_{X}^{\mathrm{TVag}}<1$. An opinion has monovagueness when only a single composite value has (vague) belief mass assigned to it. On the other hand, an opinion has pluri-vagueness when several composite values have (vague) belief mass assigned to them.

Note that there is the difference between belief vacuity represented as uncertainty mass $u_{X}$ and belief vagueness in SL. Uncertainty mass reflects vacuity of evidence, whereas vagueness results from evidence that fails to discriminate between specific singletons. A totally vacuous opinion - by definition - does not contain any vagueness. Hyper-opinions can contain vagueness, whereas multinomial and binomial opinions never contain vagueness. The ability to express vagueness is thus the main aspect that makes hyper-opinions different from multinomial and binomial opinions.

Under the assumption that collected evidence never decays, vacuity can only decrease over time, because accumulated evidence is never lost. As the natural complement, singular and vague belief mass can only increase. At the extreme, a dogmatic opinion where $b_{X}^{\text {TVag }}=1$ expresses dogmatic vagueness. A dogmatic opinion where $b_{X}^{\mathrm{TS} i n}=1$ expresses dogmatic singular belief, which is equivalent to a traditional probability distribution over a random variable.

The base rate distribution has an influence on the interpretation of belief vagueness in terms of 'sharp' or 'blunt' belief vagueness. This gives rise to the definitions of sharp and blunt vagueness defined below.

Definition 4 (Sharp Vagueness): Let $\mathbb{X}$ be a domain with variable $X$, and let $\omega_{X}$ be an opinion on $\mathbb{X}$. The sharp vagueness contained in the opinion $\omega_{X}$ is the function $b_{X}^{\text {SVag }}: \mathscr{C}(\mathbb{X}) \rightarrow[0,1]$ expressed as:

$$
\text { Sharp vagueness: } \quad b_{X}^{\mathrm{SVag}}=\sum_{x \in \mathscr{C}(\mathbb{X})}\left(1-\boldsymbol{a}_{X}(x)\right) \boldsymbol{b}_{X}(x) .
$$

Definition 5 (Blunt Vagueness): Let $\mathbb{X}$ be a domain with variable $X$, and let $\omega_{X}$ be an opinion on $\mathbb{X}$. The blunt vagueness contained in the opinion $\omega_{X}$ is the function $b_{X}^{\mathrm{BVag}}: \mathscr{C}(\mathbb{X}) \rightarrow[0,1]$ expressed as:

$$
\text { Blunt vagueness: } b_{X}^{\mathrm{BVag}}=\sum_{x \in \mathscr{C}(\mathbb{X})} \boldsymbol{a}_{X}(x) \boldsymbol{b}_{X}(x) .
$$

It can be verified that $b_{X}^{\mathrm{TVag}}=b_{X}^{\mathrm{SVag}}+b_{X}^{\mathrm{BVag}}$. 
Sharp vagueness can be interpreted as singular belief mass. Similarly, blunt vagueness can be interpreted as vacuity. Following that reasoning we define sharp singular belief mass $b_{X}^{\mathrm{SS} i n}$ and blunt vacuity $u_{X}^{\mathrm{BVac}}$ as:

$$
\begin{array}{ll}
\text { Sharp singular belief: } & b_{X}^{\mathrm{SSin}}=b_{X}^{\mathrm{TSin}}+b_{X}^{\mathrm{SVag}} \\
\text { Blunt vacuity: } & u_{X}^{\mathrm{BVac}}=u_{X}^{\mathrm{Vac}}+b_{X}^{\mathrm{BVag}}
\end{array}
$$

A relatively small base rate of a composite state value corresponds to a relatively sharp vague belief mass which can be interpreted as singular belief mass. In the opposite case, a relatively large base rate of a composite state value corresponds to a relatively blunt vague belief mass which can be interpreted as vacuity. At the extreme, belief mass assigned to a composite value $x$ where $\boldsymbol{a}_{X}(x)=1$ would be equivalent to vacuity.

\section{Belief Vacuity}

As discussed in Section II-A, belief vacuity results from the lack of evidence or knowledge, and is represented as 'uncertainty mass' in SL. This type of uncertainty means that the analyst is uncertain with regard to the truth of any state value in the domain, due to the lack of evidence to support any particular state value. Vacuity also emerges when the analyst does not understand the dynamics and causal relationships of the situation, making it impossible to determine its actual state or predict its future state.

In SL, belief vacuity is represented as uncertainty mass in all three types of opinions (i.e., binomial, multinomial, and hyperopinions). Uncertainty mass results from the lack of evidence, which is the same as vacuity of belief. General background knowledge about a particular state value $x$ is represented as base rate (or aka prior belief), denoted by $\boldsymbol{a}_{X}(x)$, which is mainly used to interpret the uncertainty mass when deriving the projected probability distribution over a set of state values. Therefore, when the base rate $\boldsymbol{a}_{X}(x)$ is known, the amount of uncertainty caused by vacuity can be well interpreted for efficient decision making [2].

Belief vacuity is simply the traditional uncertainty mass of subjective opinions, usually denoted by $u_{X}$ in the case of an opinion $\omega_{X}=\left(\boldsymbol{b}_{X}, u_{X}, \boldsymbol{a}_{X}\right)$.

As a simple example of a binomial opinion in SL, an opinion set $(0,0,1, a)$ is totally vacuous because the uncertainty mass $u=1$ excludes belief support for any of the state values.

It can be shown that there is additivity between total singular belief, total vague belief and vacuity of a hyper-opinion:

$$
b_{X}^{\mathrm{TSin}}+b_{X}^{\mathrm{TVag}}+u_{X}^{\mathrm{Vac}}=1 .
$$

We denote by 'HBBU point' (Hyper Basic Belief Uncertainty) the point of a hyper-opinion defined by Eq. (25) in a barycentric coordinate system, as illustrated in Fig. 1.

When the hyper-opinion is projected to a multinomial opinion by removing the vagueness component, the multinomial additivity of Eq. (26) emerges:

$$
b_{X}^{\mathrm{SSin}}+u_{X}^{B V a c}=1 .
$$

We denote by 'MBBU point' (Multinomial Basic Belief Uncertainty) the point of a multinomial opinion defined by Eq. (26) in a barycentric coordinate system, as illustrated in Fig. 1. The MBBU point can result from the projection of the HBBU point of a hyper-opinion when projecting it to a multinomial opinion.

\section{Belief Dissonance}

As explained in Section II-A, belief dissonance occurs when available evidence supports separate non-overlapping state values or subsets of the domain.

1) Dissonance in Binomial or Multinomial Opinions: In binomial or multinomial opinions, a domain consists of singletons. The belief dissonance of an opinion is measured based on how much belief supports singleton state values. For example, given a binomial opinion with $(b, d, u, a)=(0.5,0.5,0, a)$ based on Eq. (5), although uncertainty is zero, one cannot make a decision due to the same amount of belief supporting two separate state values. This kind of 'inconclusiveness' in a decision making situation is considered in the concept of belief dissonance in SL. Similarly, in a multinomial opinion where each singleton can be supported by some belief mass, the opinion's belief dissonance can be measured based on the sum of belief masses. Given a multinomial opinion, the measure of dissonance can be represented by:

$$
\dot{b}_{X}^{\text {Diss }}=\sum_{x_{i} \in \mathbb{X}}\left(\frac{\boldsymbol{b}_{X}\left(x_{i}\right) \sum_{x_{j} \in \mathbb{X} \backslash x_{i}} \boldsymbol{b}_{X}\left(x_{j}\right) \operatorname{Bal}\left(x_{j}, x_{i}\right)}{\sum_{x_{j} \in \mathbb{X} \backslash x_{i}} \boldsymbol{b}_{X}\left(x_{j}\right)}\right),
$$

where the relative mass balance between a pair of belief masses $\boldsymbol{b}_{X}\left(x_{j}\right)$ and $\boldsymbol{b}_{X}\left(x_{i}\right)$ is expressed by:

$$
\operatorname{Bal}\left(x_{j}, x_{i}\right)=1-\frac{\left|\boldsymbol{b}_{X}\left(x_{j}\right)-\boldsymbol{b}_{X}\left(x_{i}\right)\right|}{\boldsymbol{b}_{X}\left(x_{j}\right)+\boldsymbol{b}_{X}\left(x_{i}\right)} .
$$

The relative mass balance has its maximum at 1 when $\boldsymbol{b}_{X}\left(x_{j}\right)=\boldsymbol{b}_{X}\left(x_{i}\right)$. The relative mass balance has the minimum at 0 when one of the belief masses equals 0 .

In case of zero sum of belief masses in both the nominators and the denominators of Eqs. (27)-(31), it must be assumed that the fraction of sums of belief masses equals 1 .

2) Dissonance in Hyper-opinions: Hyper-opinions have belief mass on composite state values $x \in \mathscr{R}(\mathbb{X})$, i.e., there can be belief mass assigned to partially overlapping composite values, which does not represent a dissonant belief.

Assume a domain $\mathbb{X}$ with a hyper-opinion $\omega_{X}$. The amount of belief dissonance in $\omega_{X}$ is given by:

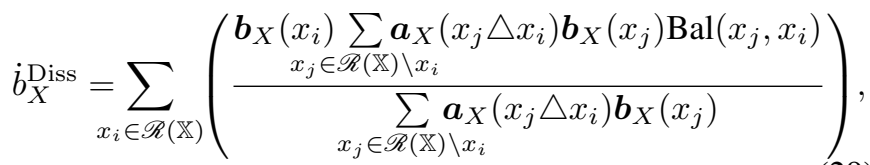

where $\boldsymbol{a}_{X}\left(x_{j} \triangle x_{i}\right)$ denotes the base rate of the symmetric difference between $x_{j}$ and $x_{i}$ which thereby represent the base rate of the parts of $x_{j}$ and $x_{i}$ that are in 'dissonance' with each 
other. As before, $\operatorname{Bal}\left(x_{j}, x_{i}\right)$ denotes the relative mass balance between a pair of belief masses as expressed by Eq. (28).

\section{E. Belief Consonance}

Belief consonance occurs when available evidence supports composite state values that are contained within each other, i.e., when there is belief mass on both a larger state value and on a smaller subset fully contained within the larger value. Belief consonance only exists for hyper-opinions because these apply to composite state values, and is given by:

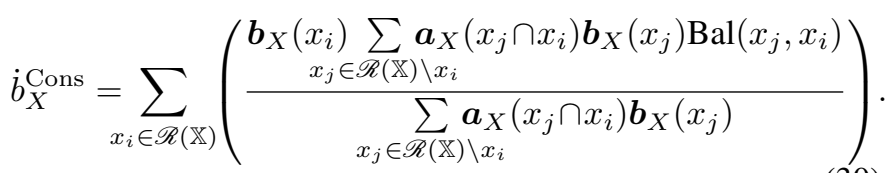

\section{F. Monosonance}

As explained in Section II-A, belief monosonance occurs when belief mass (mainly) supports a single state value, and is given by:

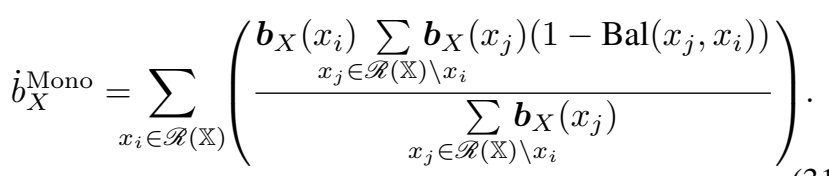

The interpretation of belief monosonance is that it increases as a function of the relative weight imbalance between two belief masses $\boldsymbol{b}_{X}\left(x_{i}\right)$ and $\boldsymbol{b}_{X}\left(x_{j}\right)$. In the case of two disjoint state values $x_{i}$ and $x_{j}$ where $\boldsymbol{b}_{X}\left(x_{i}\right) \gg 0$, then their relative monosonance approaches $\boldsymbol{b}_{X}\left(x_{i}\right)$ when $\boldsymbol{b}_{X}\left(x_{j}\right) \longrightarrow 0$.

In general, it can be observed that belief dissonance, belief consonance and belief monosonance, which are the key belief masses associated with Intra-Belief Uncertainty (IBU), increase when vacuity decreases. In order to have them perfectly fit to 1 when they are summed up with the vacuity uncertainty mass (see Eq. (34)), they need to be normalized with the factor $\nu^{\mathrm{IBU}}$ defined as:

$$
\nu^{\mathrm{IBU}}=\frac{\dot{b}_{X}^{\mathrm{Diss}}+\dot{b}_{X}^{\mathrm{Cons}}+\dot{b}_{X}^{\mathrm{Mono}}}{1-u_{X}^{\mathrm{Vac}}} .
$$

Then the normalized IBU characteristics are defined as:

$$
b_{X}^{\text {Diss }}=\frac{\dot{b}_{X}^{\text {Diss }}}{\nu^{\mathrm{IBU}}}, \quad b_{X}^{\text {Cons }}=\frac{\dot{b}_{X}^{\text {Cons }}}{\nu^{\mathrm{IBU}}}, \quad b_{X}^{\mathrm{Mono}}=\frac{\dot{b}_{X}^{\text {Mono }}}{\nu^{\mathrm{IBU}}} .
$$

Given the normalized IBU characteristics, additivity is expressed by Eq.(34).

$$
b_{X}^{\text {Diss }}+b_{X}^{\text {Cons }}+b_{X}^{\mathrm{Mono}}+u_{X}^{\mathrm{Vac}}=1 .
$$

We denote the point defined by Eq. (34) by 'IBU point' in a barycentric coordinate system, as illustrated in Fig. 1.

\section{EXAMPLE AND VISUALIZATION}

The uncertainty characteristics defined in the sections above can be visualized for any opinion. For the purpose of illustration, we assume as an example the hyper-opinion defined on a ternary domain $\mathbb{X}=\left\{x_{1}, x_{2}, x_{3}\right\}$, where the belief mass and base rate distributions are given in Table I. Note the compact notation, e.g., $\left\{x_{1}, x_{2}\right\}$, is denoted as $x_{1,2}$.

TABLE I: Trinomial Opinion

\begin{tabular}{|c|c|c|c|c|c|}
\hline \multicolumn{2}{|c|}{$\begin{array}{c}\text { Basic } \\
\text { Belief Uncertainty }\end{array}$} & \multicolumn{2}{|c|}{$\begin{array}{c}\text { Sharpness and } \\
\text { Bluntness }\end{array}$} & \multicolumn{2}{|c|}{$\begin{array}{l}\text { Intra-Belief } \\
\text { Uncertainty }\end{array}$} \\
\hline $\begin{array}{l}b_{X}^{\mathrm{TSin}} \\
b_{X}^{\mathrm{TVag}} \\
u_{X}^{\mathrm{Vac}}\end{array}$ & $\begin{array}{l}=0.200 \\
=0.600 \\
=0.200\end{array}$ & $\begin{array}{l}b_{X}^{\text {SVag }} \\
b_{X}^{\text {BVag }} \\
b_{X}^{\text {SSin }} \\
u_{X}^{\text {BVac }}\end{array}$ & $\begin{array}{l}=0.120 \\
=0.480 \\
=0.320 \\
=0.680\end{array}$ & $\begin{array}{l}b_{X}^{\text {Diss }} \\
b_{X}^{\text {Cons }} \\
b_{X}^{\text {Mono }}\end{array}$ & $\begin{array}{l}=0.203 \\
=0.175 \\
=0.422\end{array}$ \\
\hline
\end{tabular}

\begin{tabular}{|lll|lll|l|}
\hline Value & $\boldsymbol{b}_{X}(\cdot)$ & $\boldsymbol{a}_{X}(\cdot)$ & Value & $\boldsymbol{b}_{X}(\cdot)$ & $\boldsymbol{a}_{X}(\cdot)$ & Vacuity \\
\hline & & & & & & \multirow{2}{*}{ Vac $=0.2$} \\
$x_{1}$ & 0.10 & 0.50 & $x_{1,2}$ & 0.60 & 0.80 & $u_{X}$ \\
$x_{2}$ & 0.10 & 0.30 & $x_{1,3}$ & 0.00 & 0.70 & \\
$x_{3}$ & 0.00 & 0.20 & $x_{2,3}$ & 0.00 & 0.50 & \\
\hline
\end{tabular}

From the above opinion, the uncertainty characteristics are given in Table II.

TABLE II: Uncertainty Characteristics

The uncertainty characteristics of Table II are visualized in Fig. 1. At the top the HBBU (Hypernomial Basic Belief Uncertainty) point and MBBU (Multinomial Basic Belief Uncertainty) point are shown in a triangular barycentric coordinate system. At the bottom, the IBU (Intra-Belief Uncertainty) point is visualized in a triangular barycentric coordinate system where the size of the coordinate system shrinks as a function of the belief vacuity $u_{X}^{\mathrm{Vac}}$, which reflects the additivity of Eq. (34).

\section{Discussions}

The general concept of uncertainty is complex due to its multidimensional nature and causes derived from its fundamental complexity. In order to obtain a greater understanding of the various dimensions of uncertainty, we define two separate categories of uncertainty called basic belief uncertainty (consisting of singularity, vagueness and vacuity) and intrabelief uncertainty (consisting of dissonance, consonance, and monosonance). These categories have their own internal structure in terms of additivity. The differences between categories can be still quite subtle because it would be quite challenging to distinguish between vagueness and dissonance in some contexts where it can be difficult to judge whether there is vagueness (i.e., confusing evidence on separate singletons together) or dissonance (i.e., separate evidence on each singleton). In general, vagueness refers to confusion because an observer cannot clearly observe a singleton state value due to the the evidence being non-discriminating, while dissonance 


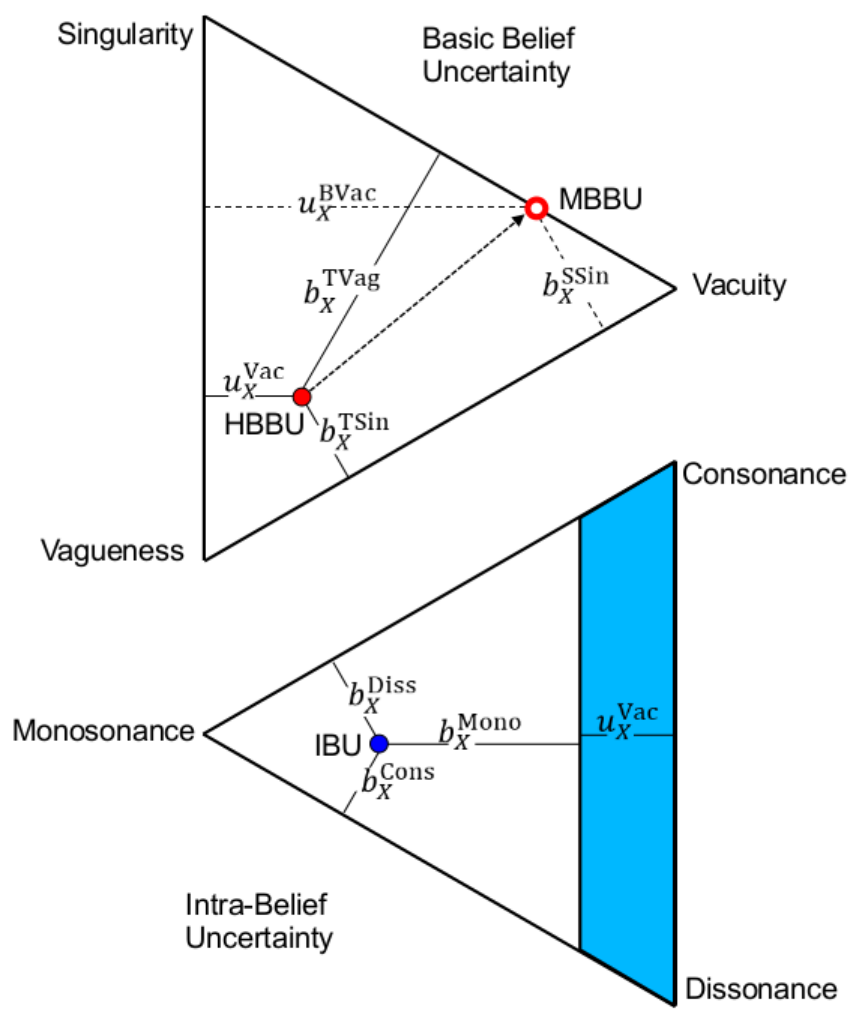

Fig. 1: Visualization of uncertainty characteristics.

derives from conflicting observations which can be caused by sensor malfunction or compromise (i.e., observation error or adversarial manipulation) or dynamic states of a situation (i.e., perfect observations but actually the situation itself shows different observations at different times). Regardless of the causes of the conflicting observations reported, the dissonance can lead an opinion being polarized into dissonant belief which makes decision making hard due to inconclusiveness based on evidence supporting different state values.

\section{CONCLUSION \& Future Work}

In this work, we investigated multidimensional aspects of uncertainty based on its root causes within the structure of subjective opinions. In particular, we explained how the various types of uncertainty can be derived and be interpreted. To be specific, we dissected the traditional representation of opinions by considering various uncertainty characteristics that were defined in terms of the concepts of the 'singularity', 'vagueness', 'vacuity', 'dissonance', 'consonance' and 'monosonance' of an opinion. We defined those concepts in the formalism of SL and showed how these characteristics can be manifested in the three different opinion classes which are binomial, multinomial, and hyper-opinions. We proposed a visualization of these uncertainty characteristics in order to facilitate easier interpretation for effective decision making.
As the future work direction, we plan to conduct a simulation study in order to investigate how the uncertainty characteristics in subjective opinions can affect decision making performance in various environmental conditions such as under varying the degree of compromised observations, base rates of possible outcomes, and the degrees of consonance, dissonance, and monosonance of given opinions.

\section{REFERENCES}

[1] M. Brugnach, A. Dewulf, C. Pahl-Wostl, and T. Taillieu, "Toward a relational concept of uncertainty: About knowing too little, knowing too differently, and accepting not to know," Ecology and Society, vol. 13, no. 2, p. 30, 2008.

[2] J. Cho, T. Cook, S. Rager, J. O'Donovan, and S. Adal1, "Modeling and analysis of uncertainty-based false information propagation in social networks," in IEEE Global Communications Conference (GLOBECOM 2017), Singapore, Dec. 2017.

[3] D. Dubois and H. Prade, Possibility theory: an approach to computerized processing of uncertainty. Springer Science \& Business Media, 2012.

[4] S. E. Fienberg et al., "When did bayesian inference become 'bayesian'?" Bayesian Analysis, vol. 1, no. 1, pp. 1-40, 2006.

[5] R. K. Hankin, "A generalization of the Dirichlet distribution," Journal of Statistical Software, vol. 33, no. 11, pp. 1-18, February 2010.

[6] B. Huang, A. Kimmig, L. Getoor, and J. Golbeck, "Probabilistic soft logic for trust analysis in social networks," in International Workshop on Statistical Relational AI, 2012, pp. 1-8.

[7] A. Jøsang, Subjective Logic: A Formalism for Reasoning Under Uncertainty. Springer, Heidelberg, 2016.

[8] A. Kimmig, S. Bach, M. Broecheler, B. Huang, and L. Getoor, "A short introduction to probabilistic soft logic," in Proceedings of the NIPS Workshop on Probabilistic Programming: Foundations and Applications, 2012, pp. 1-4.

[9] A. D. Kiureghian and O. Ditlevsen, "Aleatory or epistemic? does it matter?" Structural Safety, vol. 31, no. 2, pp. 105-112, 2009, risk Acceptance and Risk Communication.

[10] R. B. Myerson, Game Theory. Harvard university press, 2013.

[11] G. Shafer, A Mathematical Theory of Evidence. Princeton University Press, 1976.

[12] F. Smarandache and J. Dezert, Advances and Applications of DSmT for Information Fusion (Collected works), second volume: Collected Works. Infinite Study, 2006, vol. 2.

[13] P. Smets and R. Kennes, "The transferable belief model," Artificial Intelligence, vol. 66, pp. 191-234, 1994.

[14] D. Tuggy, "Ambiguity, polysemy, and vagueness," Cognitive Linguistics, vol. 4, no. 3, pp. 273-290, 2009.

[15] A. Tversky and D. Kahneman, "The framing of decisions and the psychology of choice," in Environmental Impact Assessment, Technology Assessment, and Risk Analysis. Springer, 1985, pp. 107-129.

[16] W. E. Walker, P. Harremoës, J. Rotmans, J. P. van der Sluijs, M. B. van Asselt, P. Janssen, and M. P. Krayer von Krauss, "Defining uncertainty: a conceptual basis for uncertainty management in model-based decision support," Integrated Assessment, vol. 4, no. 1, pp. 5-17, 2003.

[17] Y. Wang and M. P. Singh, "Evidence-based trust: A mathematical model geared for multiagent systems," ACM Transactions on Autonomous and Adaptive Systems (TAAS), vol. 5, no. 4, p. 14, 2010.

[18] L. A. Zadeh, "Fuzzy sets," Information and Control, vol. 8, no. 3, pp. 338-353, 1965. 